\title{
Denseness of Norm-Attaining Mappings on Banach Spaces
}

\author{
by \\ Yun Sung Choi, Han Ju LeE and Hyun Gwi Song
}

\begin{abstract}
Let $X$ and $Y$ be Banach spaces. Let $P\left({ }^{n} X: Y\right)$ be the space of all $Y$-valued continuous $n$-homogeneous polynomials on $X$. We show that the set of all norm-attaining elements is dense in $P\left({ }^{n} X: Y\right)$ when a set of u.s.e. points of the unit ball $B_{X}$ is dense in the unit sphere $S_{X}$. Applying strong peak points instead of u.s.e. points, we generalize this result to a closed subspace of $C_{b}(M, Y)$, where $M$ is a complete metric space. For complex Banach spaces $X$ and $Y$, let $A_{b}\left(B_{X}: Y\right)$ be the Banach space of all bounded continuous $Y$-valued mappings $f$ on $B_{X}$ whose restrictions $\left.f\right|_{B_{X}^{\circ}}$ to the open unit ball are holomorphic. It follows that the set of all norm-attaining elements is dense in $A_{b}\left(B_{X}: Y\right)$ if the set of all strong peak points in $A_{b}\left(B_{X}\right)$ is a norming subset for $A_{b}\left(B_{X}\right)$.
\end{abstract}

2010 Mathematics Subject Classification: 46B04, 46G20, 46G25, 46B22.

Keywords: homogeneous polynomial, norm-attaining element, uniformly strongly exposed point, strong peak point.

\section{§1. Introduction}

Let $X, Y$ be Banach spaces over a scalar field $\mathbb{F}$, where $\mathbb{F}$ is the real or complex field. The celebrated Bishop-Phelps theorem [6] says that the set of all norm-attaining continuous linear functionals is dense in the dual space $X^{*}$. Motivated by the Bishop-Phelps theorem, Lindenstrauss [20] studied the denseness of the normattaining operators in the space $L(X, Y)$ of all continuous linear operators. Recall that an operator $T \in L(X, Y)$ is said to attain its norm if $\|T\|=\left\|T\left(x_{0}\right)\right\|$ for some $x_{0} \in B_{X}$, where $B_{X}$ is the unit ball of $X$. In [20, Proposition 1] he showed that if

Communicated by H. Okamoto. Received April 18, 2009. Revised August 10, 2009.

Y. S. Choi: Department of Mathematics, POSTECH, Republic of Korea;

e-mail: mathchoi@postech.ac.kr

H. J. Lee (corresponding author): Department of Mathematics Education, Dongguk University - Seoul, Republic of Korea;

e-mail: hanjulee@dongguk.edu

H. G. Song: Department of Mathematics, Sogang University, Republic of Korea;

e-mail: hyuns@postech.ac.kr

(c) 2010 Research Institute for Mathematical Sciences, Kyoto University. All rights reserved. 
$B_{X}$ is the closed convex hull of a set of uniformly strongly exposed (u.s.e.) points, then $X$ has property $A$, that is, for every Banach space $Y$, the set of norm-attaining elements is dense in $L(X, Y)$. Payá and Saleh [22] extended this result to $n$-linear forms on $X$, and showed that if $B_{X}$ is the closed absolutely convex hull of a set of u.s.e. points, then the set of all norm-attaining elements is dense in $L\left({ }^{n} X\right)$, the Banach space of all bounded $n$-linear forms on $X$. Notice that if $B_{X}$ is the closed absolutely convex hull of a set $E$ of u.s.e. points, then $E$ is a norming set for $L(X, Y)$.

We shall study a similar question for the space $P\left({ }^{n} X: Y\right)$ of all continuous $n$-homogeneous polynomials from $X$ into $Y$. In particular, if a set of u.s.e. points on $S_{X}$ is a norming set for $P\left({ }^{n} X: Y\right)$, then the set of norm-attaining elements is dense in $P\left({ }^{n} X: Y\right)$. Applying strong peak points instead of u.s.e. points, we shall generalize this result to a closed subspace of $C_{b}(M, Y)$, where $M$ is a complete metric space and $C_{b}(M, Y)$ is the Banach space of all bounded continuous mappings from $X$ to $Y$ with the norm $\|f\|=\sup \{\|f(t)\|: t \in M\}$.

On the other hand, Lindenstrauss [20. Theorem 1] proved that the set of all bounded linear operators of $X$ into $Y$ whose second adjoints attain their norms is dense in $L(X, Y)$. In 1996 Acosta [2] extended this result to continuous bilinear forms, and in 2002 Aron, Garcia and Maestre [5] showed that this is also true for scalar-valued continuous 2-homogeneous polynomials. Recently, Acosta, Garcia and Maestre [4] extended it to $n$-linear mappings. We shall extend the result of [5] to the vector-valued case by modifying their proof, which is originally based on that of Lindenstrauss. Finally, we give a necessary condition for a complex Banach space to have property $A$.

For completeness we recall some terminology. A continuous $n$-homogeneous polynomial $P$ from $X$ to $Y$ is a mapping $P: X \rightarrow Y$ defined by $P(x)=L(x, \ldots, x)$, where $L$ is a continuous $n$-linear mapping from $X \overbrace{\times \cdots \times}^{n} X \rightarrow Y$. The space of all continuous $n$-homogeneous polynomials from $X$ to $Y$ is denoted by $P\left({ }^{n} X: Y\right)$ and it is a Banach space when equipped with the norm $\|P\|=\sup \left\{\|P(x)\|: x \in B_{X}\right\}$. An $n$-homogeneous polynomial $P$ is said to attain its norm if $\|P\|=\left\|P\left(x_{0}\right)\right\|$ for some $x_{0} \in B_{X}$. It is clear that $P\left({ }^{n} X: Y\right)$ is a closed subspace of $C_{b}\left(B_{X}: Y\right)$. For the scalar-valued case we denote the former spaces simply by $P\left({ }^{n} X\right)$ or $C_{b}(M)$. When $A$ is a subspace of $C_{b}(M: Y)$, a subset $\Gamma$ of $M$ is said to be a norming set for $A$ if $\|f\|=\sup \{\|f(t)\|: t \in \Gamma\}$ for each $f \in A$. It is easy to see that if $E \subset B_{X}$ is a norming set for $P\left({ }^{n} X\right)$, then it is also a norming set for $P\left({ }^{n} X: Y\right)$ for every Banach space $Y$.

An element $x \in B_{X}$ is said to be a strongly exposed point for $B_{X}$ if there is a linear functional $f \in B_{X^{*}}$ such that $f(x)=1$ and whenever there is a sequence $\left\{x_{n}\right\}_{n=1}^{\infty}$ in $B_{X}$ satisfying $\lim _{n} \operatorname{Re} f\left(x_{n}\right)=1$, we get $\lim _{n}\left\|x_{n}-x\right\|=0$. A set 
$\left\{x_{\alpha}\right\}_{\alpha}$ of points on $S_{X}$ is called uniformly strongly exposed (u.s.e.) if there are a function $\delta(\epsilon)$ with $\delta(\epsilon)>0$ for every $\epsilon>0$, and a set $\left\{f_{\alpha}\right\}_{\alpha}$ of elements of norm 1 in $X^{*}$ such that for every $\alpha, f_{\alpha}\left(x_{\alpha}\right)=1$, and for any $x$,

$$
\|x\| \leq 1 \text { and } \operatorname{Re} f_{\alpha}(x) \geq 1-\delta(\epsilon) \text { imply }\left\|x-x_{\alpha}\right\| \leq \epsilon .
$$

In this case we say that $\left\{f_{\alpha}\right\}$ uniformly strongly exposes $\left\{x_{\alpha}\right\}$.

A nonzero function $f \in C_{b}(M: Y)$ is said to be a strong peak function at $t$ if whenever there is a sequence $\left\{t_{n}\right\}_{n}$ in $M$ with $\lim _{n}\left\|f\left(t_{n}\right)\right\|=\|f\|$, we get $\lim _{n} t_{n}=t$. Such a point $t$ is said to be a strong peak point of $f$. When $A$ is a subspace of $C_{b}(M: Y)$, we set $\rho A=\{t: t$ is a strong peak point of some $f \in A\}$.

\section{§2. Main results}

Theorem 2.1. Let $X$ and $Y$ be Banach spaces and $n \in \mathbb{N}$. Suppose that a set $E$ of u.s.e. points on $S_{X}$ is a norming subset of $P\left({ }^{n} X\right)$. Then the set of all normattaining elements is dense in $P\left({ }^{n} X: Y\right)$.

Proof. Suppose that a set $E$ of u.s.e. points on $S_{X}$ is a norming subset of $P\left({ }^{n} X\right)$. Let $P \in P\left({ }^{n} X: Y\right),\|P\|=1$, and $0<\epsilon<1 / 3$ be given. We first choose a decreasing sequence $\left\{\epsilon_{k}\right\}$ of positive numbers so that

$$
4 \sum_{i=1}^{\infty} \epsilon_{i}<\epsilon<\frac{1}{3}, \quad 4 \sum_{i=k+1}^{\infty} \epsilon_{i}<\epsilon_{k}^{2}, \quad \epsilon_{k}<\frac{1}{10 k}, \quad k=1,2, \ldots
$$

Using induction, we next choose sequences $\left\{P_{k}\right\}_{k=1}^{\infty}$ in $\mathcal{P}\left({ }^{n} X: Y\right),\left\{x_{k}\right\}_{k=1}^{\infty}$ in $E$ and $\left\{x_{k}^{*}\right\}_{k=1}^{\infty}$ in $S_{X^{*}}$ so that

$$
\begin{aligned}
& P_{1}=P, \\
& \left\|P_{k}\left(x_{k}\right)\right\| \geq\left\|P_{k}\right\|-\epsilon_{k}^{2} \quad \text { and } \quad\left\|x_{k}\right\|=1, \quad x_{k}^{*}\left(x_{k}\right)=1,
\end{aligned}
$$

where $\left\{x_{k}^{*}\right\}$ uniformly strongly exposes $\left\{x_{k}\right\}$,

$$
P_{k+1}(x)=P_{k}(x)+\epsilon_{k}\left(x_{k}^{*}(x)\right)^{n} P_{k}\left(x_{k}\right) \quad(x \in X) .
$$

Having chosen these sequences, we see that the following hold:

$$
\begin{aligned}
& \left\|P_{j}-P_{k}\right\| \leq \frac{4}{3} \sum_{i=j}^{k-1} \epsilon_{i}, \quad\left\|P_{k}\right\| \leq \frac{4}{3}, \quad j<k, \\
& \left\|P_{k+1}\right\| \geq\left\|P_{k}\right\|+\epsilon_{k}\left\|P_{k}\right\|-\epsilon_{k}^{2}-\epsilon_{k}^{3}, \\
& \left\|P_{k+1}\right\| \leq\left\|P_{k}\right\|+\epsilon_{k}\left|x_{k}^{*}\left(x_{l}\right)\right|^{n}\left\|P_{k}\right\|+\epsilon_{k}^{2}+2 \cdot \frac{4}{3} \sum_{i=k+1}^{l-1} \epsilon_{i}, \quad k+1<l .
\end{aligned}
$$


The assertion (2.5) can be easily proved by induction and 2.6 follows directly from 2.3) and 2.4. To see 2.7), for $k+1<l$ we have

$$
\begin{aligned}
\left\|P_{k+1}\right\| & \leq\left\|P_{l}\right\|+\left\|P_{k+1}-P_{l}\right\| \leq\left\|P_{l}\left(x_{l}\right)\right\|+\epsilon_{l}^{2}+\frac{4}{3} \sum_{i=k+1}^{l-1} \epsilon_{i} \\
& \leq\left\|P_{k}\left(x_{l}\right)\right\|+\epsilon_{k}\left|x_{k}^{*}\left(x_{l}\right)\right|^{n}\left\|P_{k}\right\|+\epsilon_{k}^{2}+2 \cdot \frac{4}{3} \sum_{i=k+1}^{l-1} \epsilon_{i} \\
& \leq\left\|P_{k}\right\|+\epsilon_{k}\left|x_{k}^{*}\left(x_{l}\right)\right|^{n}\left\|P_{k}\right\|+\epsilon_{k}^{2}+2 \cdot \frac{4}{3} \sum_{i=k+1}^{l-1} \epsilon_{i} .
\end{aligned}
$$

By $(2.5)$, the sequence $\left\{P_{k}\right\}$ converges in the norm topology to $Q \in P\left({ }^{n} X: Y\right)$ satisfying $\|P-Q\|<\epsilon$.

By 2.6 and 2.7 we have, for every $l>k+1$,

$$
\epsilon_{k}\left\|P_{k}\right\|-\epsilon_{k}^{2}-\epsilon_{k}^{3} \leq \epsilon_{k}\left|x_{k}^{*}\left(x_{l}\right)\right|^{n}\left\|P_{k}\right\|+2 \epsilon_{k}^{2},
$$

and hence $1-4 \epsilon_{k}<\left|x_{k}^{*}\left(x_{l}\right)\right|^{n}$ since $\left\|P_{k}\right\| \geq 1$ for each $k \geq 1$.

Since $E$ is uniformly strongly exposed, $\left\{x_{k}\right\}$ has a norm convergent subsequence to some $x_{0} \in S_{X}$ by Lemma 6 in [1]. Then we can see that $\left\|Q\left(x_{0}\right)\right\|=$ $\|Q\|$.

Now we are going to generalize this result to a closed subspace of $C_{b}(M, Y)$ with strong peak points instead of u.s.e. points.

Theorem 2.2. Let $(M, d)$ be a complete metric space, $Y$ a Banach space and $A$ a closed subspace of $C_{b}(M: Y)$. Assume that there exist a norming subset $\left\{x_{\alpha}\right\}_{\alpha} \subset M$ for $A$ and a family $\left\{\varphi_{\alpha}\right\}_{\alpha}$ of functions in $C_{b}(M)$ such that each $\varphi_{\alpha}$ is a strong peak function at $x_{\alpha}$. Assume also that $A$ contains $\varphi_{\alpha}^{n} \otimes y$ for each $y \in Y$ and $n \geq 1$. Then the set of norm-attaining elements is dense in $A$.

Proof. We may assume that $\varphi_{\alpha}\left(x_{\alpha}\right)=1$ for each $\alpha$. Let $f \in A$ with $\|f\|=1$ and $\epsilon$ with $0<\epsilon<1 / 3$ be given. We choose a decreasing sequence $\left\{\epsilon_{k}\right\}$ of positive numbers so that

$$
2 \sum_{i=1}^{\infty} \epsilon_{i}<\epsilon, \quad 2 \sum_{i=k+1}^{\infty} \epsilon_{i}<\epsilon_{k}^{2}, \quad \epsilon_{k}<\frac{1}{10 k}, \quad k=1,2, \ldots
$$


We next choose inductively sequences $\left\{f_{k}\right\}_{k=1}^{\infty},\left\{x_{\alpha_{k}}\right\}_{k=1}^{\infty}$ satisfying

$$
\begin{aligned}
& f_{1}=f, \\
& \left\|f_{k}\left(x_{\alpha_{k}}\right)\right\| \geq\left\|f_{k}\right\|-\epsilon_{k}^{2}, \\
& f_{k+1}(x)=f_{k}(x)+\epsilon_{k} \tilde{\varphi}_{\alpha_{k}}(x) \cdot f_{k}\left(x_{\alpha_{k}}\right) \quad(x \in M), \\
& \left|\tilde{\varphi}_{\alpha_{k}}(x)\right|>1-1 / k \quad \text { implies } \quad d\left(x, x_{\alpha_{k}}\right)<1 / k,
\end{aligned}
$$

where $\tilde{\varphi}_{\alpha_{j}}$ is $\varphi_{\alpha_{j}}^{n_{j}}$ for some positive integer $n_{j}$. Having chosen these sequences, we verify the following:

$$
\begin{array}{ll}
\left\|f_{j}-f_{k}\right\| \leq 2 \sum_{i=j}^{k-1} \epsilon_{i}, \quad\left\|f_{k}\right\| \leq 4 / 3, & j<k, k=2,3, \ldots, \\
\left\|f_{k+1}\right\| \geq\left\|f_{k}\right\|+\epsilon_{k}\left\|f_{k}\right\|-2 \epsilon_{k}^{2}, & k=1,2, \ldots, \\
\left\|f_{k}\right\| \geq\left\|f_{j}\right\| \geq 1, & j<k, k=2,3, \ldots, \\
\left|\tilde{\varphi}_{\alpha_{j}}\left(x_{\alpha_{k}}\right)\right|>1-1 / j, & j<k, k=2,3, \ldots
\end{array}
$$

Assertion 2.13 is easy by using induction on $k$. By 2.10 and 2.11,

$$
\begin{aligned}
\left\|f_{k+1}\right\| & \geq\left\|f_{k+1}\left(x_{\alpha_{k}}\right)\right\|=\left\|f_{k}\left(x_{\alpha_{k}}\right)\left(1+\epsilon_{k} \tilde{\varphi}_{\alpha_{k}}\left(x_{\alpha_{k}}\right)\right)\right\| \\
& =\left\|f_{k}\left(x_{\alpha_{k}}\right)\right\|\left(1+\epsilon_{k}\right) \geq\left(\left\|f_{k}\right\|-\epsilon_{k}^{2}\right)\left(1+\epsilon_{k}\right) \geq\left\|f_{k}\right\|+\epsilon_{k}\left\|f_{k}\right\|-2 \epsilon_{k}^{2},
\end{aligned}
$$

so 2.14 is proved. Therefore 2.15) is an immediate consequence of 2.9 and 2.14). For $j<k$, by the triangle inequality, 2.8, 2.10), 2.13 and 2.15), we have

$$
\begin{aligned}
\left\|f_{j+1}\left(x_{\alpha_{k}}\right)\right\| & \geq\left\|f_{k}\left(x_{\alpha_{k}}\right)\right\|-\left\|f_{k}-f_{j+1}\right\| \\
& \geq\left\|f_{k}\right\|-\epsilon_{k}^{2}-2 \sum_{i=j+1}^{k-1} \epsilon_{i} \geq\left\|f_{j+1}\right\|-2 \epsilon_{j}^{2} .
\end{aligned}
$$

Hence by 2.11 and 2.14,

$$
\begin{aligned}
\epsilon_{j}\left|\tilde{\varphi}_{\alpha_{j}}\left(x_{\alpha_{k}}\right)\right| \cdot\left\|f_{j}\right\|+\left\|f_{j}\right\| & \geq\left\|f_{j+1}\left(x_{\alpha_{k}}\right)\right\| \geq\left\|f_{j+1}\right\|-2 \epsilon_{j}^{2} \\
& \geq\left\|f_{j}\right\|+\epsilon_{j}\left\|f_{j}\right\|-4 \epsilon_{j}^{2},
\end{aligned}
$$

so that

$$
\left|\tilde{\varphi}_{\alpha_{j}}\left(x_{\alpha_{k}}\right)\right| \geq 1-4 \epsilon_{j}>1-1 / j,
$$

and this proves 2.16. Let $\hat{f} \in A$ be the limit of $\left\{f_{k}\right\}$ in the norm topology. By 2.8 and 2.13, $\|\hat{f}-f\|=\lim _{n}\left\|f_{n}-f_{1}\right\| \leq 2 \sum_{i=1}^{\infty} \epsilon_{i} \leq \epsilon$. The relations 2.12 and 2.16 mean that the sequence $\left\{x_{\alpha_{k}}\right\}$ converges to some $\tilde{x}$, and by (2.10), we have $\|\hat{f}\|=\lim _{n}\left\|f_{n}\right\|=\lim _{n}\left\|f_{n}\left(x_{\alpha_{n}}\right)\right\|=\|\hat{f}(\tilde{x})\|$. Hence $\hat{f}$ attains its norm. 
For complex Banach spaces $X$ and $Y$, consider the following closed subspaces of $C_{b}\left(B_{X}: Y\right)$ :

$$
\begin{aligned}
& A_{b}\left(B_{X}: Y\right)=\left\{f \in C_{b}\left(B_{X}: Y\right):\left.f\right|_{B_{X}^{\circ}} \text { is holomorphic }\right\}, \\
& A_{u}\left(B_{X}: Y\right)=\left\{f \in A_{b}\left(B_{X}: Y\right): f \text { is uniformly continuous on } B_{X}\right\} .
\end{aligned}
$$

We denote by $A\left(B_{X}: Y\right)$ either $A_{b}\left(B_{X}: Y\right)$ or $A_{u}\left(B_{X}: Y\right)$ and write $A\left(B_{X}\right)$ in the scalar valued case.

Recall that a Banach space is said to be locally uniformly convex if for each $x \in B_{X}$ and for each sequence $\left\{x_{n}\right\}_{n}$ in $B_{X}$ with $\lim _{n}\left\|x+x_{n}\right\|=2$, we have $\lim _{n}\left\|x_{n}-x\right\|=0$. Let $A$ be the closed linear span of the constant 1 and $X^{*}$ as a subspace of $C_{b}\left(B_{X}\right)$. Notice that if $X$ is locally uniformly convex, then every element of $S_{X}$ is a strong peak point for $A$, and clearly the set $\rho A\left(B_{X}: Y\right)$ of all strong peak points of $A\left(B_{X}: Y\right)$ is also $S_{X}$, hence a norming subset for $A\left(B_{X}: Y\right)$ for every complex Banach space $Y$. Indeed, if $x \in S_{X}$, choose $x^{*} \in S_{X^{*}}$ so that $x^{*}(x)=1$. Set $f(y)=\left(x^{*}(y)+1\right) / 2$ for $y \in B_{X}$. Then $f \in A$ and $f(x)=1$. If $\lim _{n}\left|f\left(x_{n}\right)\right|=1$ for some sequence $\left\{x_{n}\right\}$ in $B_{X}$, then $\lim _{n} x^{*}\left(x_{n}\right)=1$. Since $\left|x^{*}\left(x_{n}\right)+x^{*}(x)\right| \leq\left\|x_{n}+x\right\| \leq 2$ for every $n,\left\|x_{n}+x\right\| \rightarrow 2$ and $\left\|x_{n}-x\right\| \rightarrow 0$ as $n \rightarrow \infty$. It is also clear that every strongly exposed point for $B_{X}$ is a strong peak point for $A$.

Following [13, 15], a point $x \in S_{X}$ is said to be a complex extreme point of $B_{X}$ if for any nonzero $y \in X, \int_{0}^{2 \pi}\left\|x+e^{i \theta} y\right\|^{2} \frac{d \theta}{2 \pi}>1$. A point $x \in S_{X}$ is called a strong complex extreme point of $B_{X}$ if for each $\epsilon>0$, there is $\delta>0$ such that

$$
\inf \left\{\int_{0}^{2 \pi}\left\|x+e^{i \theta} y\right\|^{2} \frac{d \theta}{2 \pi}: y \in X,\|y\| \geq \epsilon\right\} \geq 1+\delta .
$$

The set of all complex extreme points of $B_{X}$ is denoted by $\operatorname{ext}_{\mathbb{C}}\left(B_{X}\right)$, and a complex Banach space $X$ is said to be strictly complex convex if $\operatorname{ext}_{\mathbb{C}}\left(B_{X}\right)=S_{X}$. When every point of $S_{X}$ is a strong complex extreme point of $B_{X}$, the Banach space $X$ is called locally uniformly c-convex.

It was shown in [7] that if a Banach sequence space $X$ is locally uniformly $c$-convex and order continuous, then $\rho A\left(B_{X}\right)$ is dense in $S_{X}$. Therefore, $\rho A\left(B_{X}\right)$ is a norming subset for $A\left(B_{X}: Y\right)$ for every complex Banach space $Y$. For the definition of a Banach sequence space and order continuity, see [7, 16, 21]. We also notice that if $E \subset B_{X}$ is a norming set for $A\left(B_{X}\right)$, then it is also a norming set for $A\left(B_{X}: Y\right)$ for every Banach space $Y$. By the remarks above, we get the following.

Corollary 2.1. Suppose that $X$ and $Y$ are complex Banach spaces and $\rho A\left(B_{X}\right)$ is a norming subset for $A\left(B_{X}: Y\right)$. Then the set of norm-attaining elements is 
dense in $A\left(B_{X}: Y\right)$. In particular, if $X$ is locally uniformly convex, or if it is a locally uniformly c-convex, order continuous Banach sequence space, then the set of norm-attaining elements is dense in $A\left(B_{X}: Y\right)$.

It is shown in [3] that if $X$ has the Radon-Nikodým property, then the set of norm-attaining elements is dense in $A\left(B_{X}: Y\right)$. However, the complex Banach space $c_{0}$ renormed by Day's norm is locally uniformly convex [11, 12], but it does not have the Radon-Nikodým property. In addition, it is a locally uniformly $c$ convex and order continuous Banach sequence space.

It is also worth remarking that it is shown in [9] that $\rho A\left(B_{X}\right)$ is a norming subset for $A\left(B_{X}\right)$ if $X$ has the Radon-Nikodým property. Further, very recently, it has been shown in 18 that the set of all strong peak functions is dense in $A\left(B_{X}: Y\right)$ if $\rho A\left(B_{X}\right)$ is a norming subset for $A\left(B_{X}\right)$.

Example 1. Let $\varphi: \mathbb{R} \rightarrow[0, \infty]$ be an even, convex continuous function vanishing only at zero and let $w=\{w(n)\}$ be a nonincreasing sequence of positive real numbers satisfying $\sum_{n=1}^{\infty} w(n)=\infty$. For a sequence $x=\{x(n)\}_{n=1}^{\infty}, x^{*}$ is the decreasing rearrangement of $|x|=\{|x(n)|\}_{n=1}^{\infty}$.

An Orlicz-Lorentz sequence space $\lambda_{\varphi, w}$ consists of all sequences $x=\{x(n)\}$ such that for some $\lambda>0$,

$$
\varrho_{\varphi}(\lambda x)=\sum_{n=1}^{\infty} \varphi\left(\lambda x^{*}(n)\right) w(n)<\infty,
$$

and has the norm $\|x\|=\inf \left\{\lambda>0: \varrho_{\varphi}(x / \lambda) \leq 1\right\}$. Then $\lambda_{\varphi, w}$ is a Banach sequence space. We say that the function $\varphi$ satisfies condition $\delta_{2}\left(\varphi \in \delta_{2}\right)$ if there exist $K>0$ and $u_{0}>0$ such that

$$
\varphi(2 u) \leq K \varphi(u) \quad \text { for } u \in\left[0, u_{0}\right]
$$

If $\varphi \in \delta_{2}$, then $\lambda_{\varphi, w}$ is locally uniformly $c$-convex [7] and order continuous [16]. Notice that if $\varphi(t)=|t|^{p}$ for $p \geq 1$ and $w=1$, then $\lambda_{\varphi, w}=\ell_{p}$. A characterization of the local uniform convexity of an Orlicz-Lorentz function space is given in [16, 17] and a characterization of the local uniform $c$-convexity of a complex function space is given in [19].

We now extend the result of [5] to the vector-valued case, that is, show that the set of all elements whose Aron-Berner extensions attain their norms is dense in $P\left({ }^{2} X: Y\right)$.

A continuous $n$-homogeneous polynomial $P \in P\left({ }^{n} X: Y\right)$ has an extension $\bar{P} \in P\left({ }^{n} X^{* *}, Y^{* *}\right)$ to the bidual $X^{* *}$ of $X$, which is called the Aron-Berner extension of $P$. In fact, $\bar{P}$ is defined in the following way. Let $X_{1}, \ldots, X_{n}$ be 
an arbitrary collection of Banach spaces and let $\mathcal{L}\left({ }^{n}\left(X_{1} \times \cdots \times X_{n}\right)\right)$ denote the space of bounded $n$-linear forms. Given $z_{i} \in X_{i}^{* *}, 1 \leq i \leq n$, define $\bar{z}_{i}$ from $\mathcal{L}\left({ }^{n}\left(X_{1} \times \cdots \times X_{i} \times X_{i+1}^{* *} \times \cdots \times X_{n}^{* *}\right)\right)$ to $\mathcal{L}\left({ }^{n-1}\left(X_{1} \times \cdots \times X_{i-1} \times X_{i+1}^{* *} \times \cdots \times X_{n}^{* *}\right)\right)$ by

$$
\bar{z}_{i}(T)\left(x_{1}, \ldots, x_{i-1}, x_{i+1}^{* *}, \ldots, x_{n}^{* *}\right)=\left\langle z_{i}, T\left(x_{1}, \ldots, x_{i-1}, \cdot, x_{i+1}^{* *}, \ldots, x_{n}^{* *}\right)\right\rangle,
$$

where $T\left(x_{1}, \ldots, x_{i-1}, \cdot, x_{i+1}^{* *}, \ldots, x_{n}^{* *}\right)$ is the linear functional on $X_{i}$ defined by $\cdot \mapsto T\left(x_{1}, \ldots, x_{i-1}, \cdot, x_{i+1}^{* *}, \ldots, x_{n}^{* *}\right)$ and $\left\langle z, x^{*}\right\rangle$ is the duality between $X_{i}^{* *}$ and $X_{i}^{*}$. The map $\bar{z}_{i}$ is a bounded operator with norm $\left\|z_{i}\right\|$. Now, given $T \in$ $\mathcal{L}\left({ }^{n}\left(X_{1} \times \cdots \times X_{n}\right)\right)$, define the extended $n$-linear form $\bar{T} \in \mathcal{L}\left({ }^{n}\left(X_{1}^{* *} \times \cdots \times X_{n}^{* *}\right)\right)$ by

$$
\bar{T}\left(z_{1}, \ldots, z_{n}\right):=\bar{z}_{1} \circ \ldots \circ \bar{z}_{n}(T) .
$$

For a vector-valued $n$-linear mapping $L \in \mathcal{L}\left({ }^{n}\left(X_{1} \times \cdots \times X_{n}\right), Y\right)$, define

$$
\bar{L}\left(x_{1}^{* *}, \ldots, x_{n}^{* *}\right)\left(y^{*}\right)=\overline{y^{*} \circ L}\left(x_{1}^{* *}, \ldots, x_{n}^{* *}\right),
$$

where $x_{i}^{* *} \in X_{i}^{* *}, 1 \leq i \leq n$, and $y^{*} \in Y^{*}$. Then $\bar{L} \in \mathcal{L}\left({ }^{n}\left(X_{1}^{* *} \times \cdots \times X_{n}^{* *}\right), Y^{* *}\right)$ has the same norm as $L$. Let $S \in \mathcal{L}_{s}\left({ }^{n} X: Y\right)$ be the symmetric $n$-linear mapping corresponding to $P$. Then $S$ can be extended to an $n$-linear mapping $\bar{S} \in \mathcal{L}\left({ }^{n} X^{* *}, Y^{* *}\right)$ as described above. The restriction

$$
\bar{P}(z)=\bar{S}(z, \ldots, z)
$$

is called the Aron-Berner extension of $P$. Given $z \in X^{* *}$ and $w \in Y^{*}$, we have

$$
\bar{P}(z)(w)=\overline{w \circ P}(z) .
$$

Actually this equality is often used as the definition of the vector-valued AronBerner extension based upon the scalar-valued Aron-Berner extension. Davie and Gamelin [10, Theorem 8] proved that $\|P\|=\|\bar{P}\|$. It is also worth noting that $\bar{S}$ is not symmetric in general.

Theorem 2.3. Let $X$ and $Y$ be Banach spaces. The subset of $P\left({ }^{2} X: Y\right)$ each of whose elements has the norm-attaining Aron-Berner extension is dense in $P\left({ }^{2} X: Y\right)$.

Proof. Let $P \in \mathcal{P}\left({ }^{2} X: Y\right),\|P\|=1$, and let $S$ be the symmetric bilinear mapping corresponding to $P$. Let $\epsilon$ with $0<\epsilon<1 / 4$ be given. We first choose a decreasing sequence $\left\{\epsilon_{k}\right\}$ of positive numbers which satisfies the following conditions:

$$
8 \sum_{i=1}^{\infty} \epsilon_{i}<\epsilon<\frac{1}{4}, \quad 8 \sum_{i=k+1}^{\infty} \epsilon_{i}<\epsilon_{k}^{2}, \quad \epsilon_{k}<\frac{1}{10 k}, \quad k=1,2, \ldots
$$


Using induction, we next choose sequences $\left\{P_{k}\right\}_{k=1}^{\infty}$ in $P\left({ }^{2} X: Y\right),\left\{x_{k}\right\}_{k=1}^{\infty}$ in $S_{X}$ and $\left\{f_{k}\right\}_{k=1}^{\infty}$ in $S_{Y^{*}}$ so that

$$
\begin{aligned}
& P_{1}=P, \quad\|P\|=1, \\
& f_{k}\left(P_{k}\left(x_{k}\right)\right)=\left\|P_{k}\left(x_{k}\right)\right\| \geq\left\|P_{k}\right\|-\epsilon_{k}^{2}, \\
& P_{k+1}(x)=P_{k}(x)+\epsilon_{k}\left(f_{k}\left(S_{k}\left(x_{k}, x\right)\right)\right)^{2} P_{k}\left(x_{k}\right) \quad(x \in X),
\end{aligned}
$$

where each $S_{k}$ is the symmetric bilinear mapping corresponding to $P_{k}$. Having chosen these sequences, we see that the following hold:

$$
\begin{aligned}
& \left\|P_{j}-P_{k}\right\| \leq 4\left(\frac{5}{4}\right) \sum_{i=j}^{3} \epsilon_{i}, \quad\left\|P_{k}\right\| \leq \frac{5}{4}, \quad j<k, \\
& \left\|P_{k+1}\right\| \geq\left\|P_{k}\right\|+\epsilon_{k}\left\|P_{k}\right\|^{3}-4 \epsilon_{k}^{2}, \\
& \left\|P_{j+1}\left(x_{k}\right)\right\|>\left\|P_{j+1}\right\|-2 \epsilon_{j}^{2}, \quad j<k, \\
& \left|f_{j}\left(S_{j}\left(x_{j}, x_{k}\right)\right)\right|^{2} \geq\left\|P_{j}\right\|^{2}-6 \epsilon_{j}, \quad j<k .
\end{aligned}
$$

By 2.21 and the polarization formula [14, the sequences $\left\{P_{k}\right\}$ and $\left\{S_{k}\right\}$ converge in the norm topology to $Q$ and $T$, say, respectively. Clearly $T$ is the symmetric bilinear mapping corresponding to $Q$, and $\|P-Q\|<\epsilon$.

Let $\eta>0$ be given. Then there exists $j_{0} \in \mathbb{N}$ such that

$$
\left\|Q-P_{j}\right\| \leq\left\|T-S_{j}\right\|<\eta \quad \text { for all } j \geq j_{0},
$$

hence $\left\|P_{j}\right\| \geq\|Q\|-\eta$ for all $j \geq j_{0}$.

From

$$
\left\|T-S_{j}\right\| \geq\left|f_{j}\left(T\left(x_{j}, x_{k}\right)\right)-f_{j}\left(S_{j}\left(x_{j}, x_{k}\right)\right)\right|
$$

and (2.24), we have

$$
\begin{aligned}
\left|f_{j}\left(T\left(x_{j}, x_{k}\right)\right)\right| & \geq\left|f_{j}\left(S_{j}\left(x_{j}, x_{k}\right)\right)\right|-\left\|T-S_{j}\right\| \\
& \geq \sqrt{\left\|P_{j}\right\|^{2}-6 \epsilon_{j}}-\eta \geq \sqrt{(\|Q\|-\eta)^{2}-6 \epsilon_{j}}-\eta
\end{aligned}
$$

for all $k>j \geq j_{0}$. Let $z \in X^{* *}$ be a weak-* limit point of the sequence $\left\{x_{k}\right\}$. Then for all $j \geq j_{0}$,

$$
\left\|\bar{T}\left(x_{j}, z\right)\right\| \geq \sqrt{(\|Q\|-\eta)^{2}-6 \epsilon_{j}}-\eta .
$$

Hence $\|\bar{T}(z, z)\| \geq\|Q\|-2 \eta$. Since $\eta>0$ is arbitrary, we have

$$
\|\bar{Q}(z)\|=\|\bar{T}(z, z)\| \geq\|Q\|=\|\bar{Q}\| .
$$

We finally investigate a version of Theorem 2 in [20] related to complex convexity. 
Theorem 2.4. Let $X$ be a complex Banach space with property $A$.

(1) If $X$ is isomorphic to a strictly c-convex space, then $B_{X}$ is the closed convex hull of its complex extreme points.

(2) If $X$ is isomorphic to a locally uniformly c-convex space, then $B_{X}$ is the closed convex hull of its strong complex extreme points.

Proof. We prove only (2). Let $C$ be the closed convex hull of the strong complex extreme points of $B_{X}$. Suppose that $C \neq B_{X}$. Then there are $f \in X^{*}$ with $\|f\|=1$ and $\delta, 0<\delta<1$, such that $|f(x)|<1-\delta$ for $x \in C$. Let $\|\cdot\| \mid \|$ be a locally uniformly $c$-convex norm on $X$, equivalent to the given norm $\|\cdot\|$, such that $\|x\| \leq\|x\|$ for $x \in X$. Let $Y$ be the space $X \oplus_{2} \mathbb{C}$ with the norm $\|(x, c)\|=\left(\|x\| \|^{2}+|c|^{2}\right)^{1 / 2}$. Then $Y$ is locally uniformly $c$-convex. Indeed, otherwise there exist $(x, c) \in S_{X \oplus_{2} \mathbb{C}}$, $\epsilon>0$ and a sequence $\left\{\left(x_{n}, c_{n}\right)\right\}$ such that for every $n \geq 1,\left\|\left(x_{n}, c_{n}\right)\right\| \geq \epsilon$ and

$$
\lim _{n} \int_{0}^{2 \pi}\left\|(x, c)+e^{i \theta}\left(x_{n}, c_{n}\right)\right\|^{2} \frac{d \theta}{2 \pi}=1 .
$$

Since the norm is plurisubharmonic,

$$
\begin{aligned}
1=\|x\|^{2}+|c|^{2} & \leq \int_{0}^{2 \pi}\left\|(x, c)+e^{i \theta}\left(x_{n}, c_{n}\right)\right\|^{2} \frac{d \theta}{2 \pi} \\
& =\int_{0}^{2 \pi}\left\|x+e^{i \theta} x_{n}\right\|^{2} \frac{d \theta}{2 \pi}+\int_{0}^{2 \pi}\left|c+e^{i \theta} c_{n}\right|^{2} \frac{d \theta}{2 \pi} \rightarrow 1 .
\end{aligned}
$$

So

$$
\lim _{n \rightarrow \infty} \int_{0}^{2 \pi}\left\|x+e^{i \theta} x_{n}\right\|^{2} \frac{d \theta}{2 \pi}=\|x\|^{2} \quad \text { and } \quad \lim _{n \rightarrow \infty} \int_{0}^{2 \pi}\left|c+e^{i \theta} c_{n}\right|^{2} \frac{d \theta}{2 \pi}=|c|^{2} .
$$

Since both $\left(X,\||\||)\right.$ and $\mathbb{C}$ are locally uniformly $c$-convex, we get $\lim _{n}\left\|x_{n}\right\|$ $=\lim \left|c_{n}\right|=0$, which contradicts $\inf _{n}\left\|\left(x_{n}, c_{n}\right)\right\| \geq \epsilon$.

Let $V$ be the operator from $X$ into $Y$ defined by $V x=(x, M f(x))$, where $M>2 / \delta$. Then $V$ is an (into) isomorphism and the same is true for every operator sufficiently close to $V$. We have

$$
\|V\| \geq M, \quad\|V x\| \leq\left(1+(M-2)^{2}\right)^{1 / 2} \quad \text { for } x \in C .
$$

It follows that operators sufficiently close to $V$ cannot attain their norm at a point belonging to $C$. To conclude the proof we only have to show that if $T$ is an (into) isomorphism which attains its norm at a point $x$ and if the range of $T$ is locally uniformly $c$-convex, then $x$ is a strong complex extreme point of $B_{X}$.

We may assume that $\|T x\|=\|T\|=1$. If $x$ is not a strong complex extreme point, then there are $\epsilon>0$ and a sequence $\left\{y_{n}\right\} \subset X$ such that $\left\|y_{n}\right\| \geq \epsilon$ for 
every $n$ and

$$
\lim _{n} \int_{0}^{2 \pi}\left\|x+e^{i \theta} y_{n}\right\|^{2} \frac{d \theta}{2 \pi}=1
$$

Then

$$
1 \leq \int_{0}^{2 \pi}\left\|T x+e^{i \theta} T y_{n}\right\|^{2} \frac{d \theta}{2 \pi} \leq \int_{0}^{2 \pi}\left\|x+e^{i \theta} y_{n}\right\|^{2} \frac{d \theta}{2 \pi}
$$

shows that $\left\{T y_{n}\right\}$ converges to 0 , because the range of $T$ is locally uniformly $c$-convex. Therefore, $\left\{y_{n}\right\}$ converges to 0 , which is a contradiction.

\section{Acknowledgments}

The first named author is supported by POSTECH BSRI research fund-2007. The second named author was supported by Basic Science Research Program through the National Research Foundation of Korea (NRF) funded by the Ministry of Education, Science and Technology (No. 2009-0069100).

\section{References}

[1] M. D. Acosta, Denseness of numerical radius attaining operators: renorming and embedding results, Indiana Univ. Math. J. 40 (1991), 903-914. Zbl 0725.47004 MR 1129334

[2] _ On multilinear mappings attaining their norms, Studia Math. 131 (1998), 155-165. Zbl 0934.46048 MR 1636344

[3] M. D. Acosta, J. Alaminos, D. García and M. Maestre, On holomorphic functions attaining their norms, J. Math. Anal. Appl. 297 (2004), 625-644. Zbl 1086.46034 MR 2088685

[4] M. D. Acosta, D. García and M. Maestre, A multilinear Lindenstrauss theorem, J. Funct. Anal. 235 (2006), 122-136. Zbl 1101.46029 MR 2216442

[5] R. Aron, D. García and M. Maestre, On norm-attaining polynomials, Publ. Res. Inst. Math. Sci. 39 (2003), 165-172. Zbl 1035.46005 MR 1935463

[6] E. Bishop and R. R. Phelps, A proof that every Banach space is subreflexive, Bull. Amer. Math. Soc. 67 (1961), 97-98. Zbl 0098.07905 MR 0123174

[7] Y. S. Choi, K. H. Han and H. J. Lee, Boundaries for algebras of holomorphic functions on Banach spaces, Illinois J. Math. 51 (2007), 883-896. Zbl pre05316797 MR 2379728

[8] Y. S. Choi and S. G. Kim, Norm or numerical radius attaining multilinear mappings and polynomials, J. London Math. Soc. 54 (1996), 135-147. Zbl 0858.47005 MR 1395073

[9] Y. S. Choi, H. J. Lee and H. G. Song, Bishop's theorem and differentiability of a subspace of $C_{b}(K)$, Israel J. Math., to appear.

[10] A. M. Davie and T. W. Gamelin, A theorem on polynomial-star approximation, Proc. Amer. Math. Soc. 106 (1989), 351-356. Zbl 0683.46037 MR 0947313

[11] W. J. Davis, N. Ghoussoub and J. Lindenstrauss, A lattice renorming theorem and applications to vector-valued processes, Trans. Amer. Math. Soc. 263 (1981), 531-540. Zbl 0479.46010 MR 0594424

[12] M. Day, Normed linear spaces, 3rd ed., Ergeb. Math. Grenzgeb. 21, Springer, Berlin, 1973. Zbl 0268.46013 MR 0344849

[13] S. J. Dilworth, Complex convexity and the geometry of Banach spaces, Math. Proc. Cambridge Philos. Soc. 99 (1986), 495-506. Zbl 0611.46026 MR 0830363 
[14] S. Dineen, Complex analysis on infinite-dimensional spaces, Springer, London, 1999. Zbl 1034.46504 MR 1705327

[15] P. N. Dowling, Z. Hu and D. Mupasiri, Complex convexity in Lebesgue-Bochner function spaces, Trans. Amer. Math. Soc. 348 (1996), 127-139. Zbl 0845.46018 MR 1327255

[16] P. Foralewski and P. Kolwicz, Local uniform rotundity in Calderón-Lozanovskiı̌ spaces, J. Convex Anal. 14 (2007), 395-412. Zbl 1159.46018 MR 2326095

[17] H. Hudzik, A. Kamińska and M. Mastyło, On geometric properties of Orlicz-Lorentz spaces, Canad. Math. Bull. 40 (1997), 316-329. Zbl 0903.46014 MR 1464840

[18] J. Kim and H. J. Lee, Strong peak points and strongly norm attaining points with applications to denseness and polynomial numerical indices, J. Funct. Anal. 257 (2009), 931-947. Zbl pre05586659 MR 2535458

[19] H. J. Lee, Randomized series and geometry of Banach spaces, Taiwanese J. Math., to appear.

[20] J. Lindenstrauss, On operators which attain their norm, Israel J. Math. 1 (1963), 139-148. Zbl 0127.06704 MR 0160094

[21] J. Lindenstrauss and L. Tzafriri, Classical Banach spaces II, Springer, 1979. Zbl 0403.46022 MR 0540367

[22] R. Payá and Y. Saleh, New sufficient conditions for the denseness of norm-attaining multilinear forms, Bull. London Math. Soc. 34 (2002), 212-218. Zbl 1038.46008 MR 1874249

[23] C. Stegall, Optimization and differentiation in Banach spaces, Linear Algebra Appl. 84 (1986), 191-211. Zbl 0633.46042 MR 0872283 